\title{
Heart rate and the behavioral orienting response in the rat
}

\author{
MARY NIVISON and HOLGER URSIN \\ Institute of Physiological Psychology, University of Bergen, Bergen, Norway \\ and \\ TRULS GJESTLAND \\ Electronics Laboratory, Trondheim, Norway
}

\begin{abstract}
The behavioral orienting response (OR) and heart rate (HR) were simultaneously recorded in rats in response to 85- $\mathrm{dB}$ auditory stimuli. The behavioral $\mathrm{OR}$ habituated with respect to frequency and amplitude, whereas HR responses did not fully habituate in either aspect. Most HR responses were characterized by accelerations $(\mathbf{7 6 \%}$ ) followed by decelerations $(23 \%)$. However, no differential cardiac responding with or without the behavioral OR was observed. In addition, there was no unequivocal relationship between somatic and cardiovascular responses. It is suggested that HR changes are concomitant responses rather than specific components of orienting, and that their relationship to orienting does not follow simple notions of inhibition and facilitation.
\end{abstract}

The orienting response (OR) or "investigatory reflex" was originally observed by Pavlov (1927) in animals, in the form of behavioral responses to novel stimuli. As such, the OR has two distinct components: first, an initial arrest of ongoing activity, which has been attributed to the animal's need to minimize distracting activities and to focus the relevant sensory faculties in an attempt to identify and eventually categorize the impinging stimuli; and second, a searching behavior, which has been viewed as an effort to localize the stimuli further. These responses diminish upon stimulus repetition as stimulus "novelty" diminishes (Lynn, 1966; Sokolov, 1963). The OR is easy to observe in such animals as cats (Ursin, Wester, \& Ursin, 1967) and rats (Køhler, 1976), and therefore is easy to quantify.

In addition to the $\mathrm{OR}$, man and animals react to external stimulation with two other distinct responses: defense response (DR) and startle. Of these three, only startle has been satisfactorily delineated behaviorally in relation to objective stimulus parameters. In the rat, startle is characterized by rapid, shortlasting flexor contractions that are largely dependent on auditory stimuli that reach an intensity of about $90 \mathrm{~dB}$ within $12 \mathrm{msec}$ (Hoffman \& Ison, 1980). The OR and DR seem to lack such stimulus specificity,

This research was sponsored in part by the Norwegian Research Council for Science and Humanities, and in part by the Royal Norwegian Council for Scientific and Industrial Research. The authors are grateful to Sally Coyle, Dag Hammerborg, Tove Håland, Turid Mattsson, Roar Stav, and Hege Wahl for technical assistance. M. Nivison's mailing address is: Institute of Physiological Psychology, University of Bergen, Årstadveien 21, 5000 Bergen, Norway. although the DR is presumably elicited by higher intensities.

Lacey and Lacey (1958) hypothesized that when information from the environment is accepted by the organism, as during orienting, the heart should decelerate, whereas when intense or undesirable environmental stimuli are rejected by the organism, as in a situation eliciting a defensive response, the heart should accelerate. In 1966, Graham and Clifton reviewed experimental results to date and concluded that, indeed, heart rate (HR) deceleration seemed to be the best indicator of orienting, whereas HR acceleration seemed to be the most usual response to the stimuli presumed to elicit defense. However, when attempts were made to distinguish cardiac orienting from defense, using various stimulus intensities, inconclusive data emerged. Several human studies (Germana \& Klein, 1968; Roessler, Collins, \& Burch, 1969; Uno \& Grings, 1965) failed to differentiate HR change in response to stimulus intensities ranging from above $85 \mathrm{~dB}$ to below $65 \mathrm{~dB}$. In spite of these dissenting studies, however, Graham and Slaby (1973) later suggested that this range was equivalent to the prepain zone described by Sokolov (1963), which should produce defense and long-latency HR acceleration.

The search for internal physiological indicators of observed phenomena has made the behavioral components of the OR seemingly superfluous. In view of the inconclusive evidence in the HR literature, it seems a reasonable approach to categorize the heart response in accordance with what the organism does rather than what we assume it is doing. After all, one does not orient with the heart (Obrist, Langer, 
Light, Grignolo, \& McCubbin, 1979). The actual function of $\mathrm{HR}$ change in response to external stimulation has yet to be discerned.

We have attempted to correlate the behavioral and cardiovascular response to see what, if any, response would qualify as the cardiac OR in the rat. Such a response should show differential responding with and without the behavioral $O R$, and should habituate with successive stimulus presentations.

\section{METHOD}

\section{Subjects}

A large number of animals were used in initial pilot experiments to establish the method to be used. For recording HR, first conventional wiring circuitry and later telemetric systems were tried. In the final experiment to be reported, 12 male experimentally naive Moll Wistar rats were used. They were approximately 120 days old, and they were housed individually with free access to food and water throughout the experimental period.

\section{Surgery}

Surgical procedures were performed under Mebumal (R) anesthesia at least 2 weeks before test day. Silver wires were secured to one costa (approximately the second) by a loop. Care was taken not to injure the pleura. The wire was led subcutaneously, dorsally and medially, to the scapula, and along the spine to the cranium, where it was secured to a plug fixed to the skull with dental cement and small jeweller's screws. Skin incisions were kept to a minimum, and no signs of discomfort were observed in the animals.

\section{Heart Rate Recording}

Heart beats were picked up by a Telemetric $102 \mathrm{~F}$ transmitter strapped securely to the animal's back by means of an elastic band. Electrode wires from the transmitter were inserted in the plug fixed to the skull and held in place by a screw bolt. An antenna inside the test environment and attached outside to a Dynamic 112R electric receiver picked up the heart beat signals. These and the stimulus noise were then simultaneously recorded by a Beckman R511 printer and a Hewlett-Packard 21MX computer.

\section{Apparatus}

Experimental animals were observed in the Plexiglas interior of a Grason-Stadler 1110 Skinner box. This was then placed inside a $60 \times 70 \times 50 \mathrm{~cm}$ sound-insulated box. A 3-cm-thick layer of foam rubber covered the internal walls to provide further soundproofing. A Papst 8550 system fan ensured a degree of ventilation and helped maintain the internal temperature to within $24^{\circ} \mathrm{C}$. A thermometer attached to one Plexiglas wall allowed for constant temperature monitoring. Lighting was provided by a $4.5-\mathrm{V}$ flashlight bulb. For continual monitoring via a Sony PVM-90CE video screen, a hole, $5 \mathrm{~cm}$ in diameter, was cut in the front wall of the test enclosure to accommodate a Sony CVC-21 ACE electronic viewfinder with an 8.5 -mm lens.
}

The stimulus noise was modulated white noise recorded on lownoise magnetic tapes. The noise signal was of two different types according to the modulation depth of the background noise, the most variable having fluctuations of $\pm 3 \mathrm{~dB}$ and the least variable of $\pm 1.5 \mathrm{~dB}$. Both types of background noise had an average intensity of $75 \mathrm{~dB}(\mathrm{~A})$. All stimuli were identical, with $1 \mathrm{sec}$ rise and fall times (stimulus duration equals $2 \mathrm{sec}$ ) and peak intensities of $85 \mathrm{~dB}(\mathrm{~A})$. Interstimulus intervals varied between 30 and $80 \mathrm{sec}$, and a maximum of 50 stimuli was recorded on each of the tapes. Stimulus tapes were played on a Tandberg 4-track Series 12 tape player and transmitted by a speaker from a Skinner box placed approximately $20 \mathrm{~cm}$ from one Plexiglas wall. A Bruel and Kjaer 2204 Impulse Precision sound-level meter inside the box measured the noise level throughout the test session.

\section{Experimental Procedure}

The animals were assigned randomly to one of the two stimulus tapes. On each of 3 days before test day, the animals were placed in the test environment with electrodes connected to the cranial plug and transmitter and elastic band attached. Each was then habituated to the background noise for $30 \mathrm{~min}$ per day. On the test day, the animals were placed in the test environment and habituated to the background noise for $30 \mathrm{~min}$ before the first stimulation was presented. Each animal received at least 44 and no more than 50 stimulations on the test day.

Behavior observations were made according to a slightly modified version of Køhler's (1976) scoring table for orienting responses (see Table 1). The modification consisted of the omission of ratings of ear and nose movements, since these were difficult to observe on the video screen, and the addition of scores for grooming and arrest. Behavior was rated upon stimulus presentation which was made according to a schedule of intertrial intervals. The score in each cell was then obtained by comparing poststimulus and prestimulus behavior and quantifying the change. A minimum score of 5 was necessary for a response to be called "orienting." In addition, the components of the OR were further delineated and correlated with the accompanying heart response.

Heart rate was constantly monitored by a tachiometer. However, data analysis was restricted to 50 prestimulus and 100 poststimulus beats. Trials in which interference prohibited determination of a cardiac response were deleted from the material. The large variance in prestimulus HR made it necessary to establish a criterion for calling a HR change a response. The criterion was calculated from 87 prestimulus ranges and was set at a minimum 22-beat change, in either a positive or a negative direction, from a prestimulus level. This constitutes a $6.3 \%$ change from a rat's average resting HR of 350 beats per minute (bpm). Actual mean prestimulus levels varied from animal to animal, between 310 and 378 bpm.

\section{RESULTS}

An ANOVA revealed that the number of orienting responses was not influenced by the type of background noise $[F(2,9)=.87]$. Therefore, the groups will be reported as one.

Table 1

Scoring Table Used to Quantify the Behavioral Orienting Response

\begin{tabular}{lllll}
\hline Somatic & \multicolumn{4}{c}{ Response Score } \\
\cline { 2 - 5 } Component & \multicolumn{1}{c}{1} & 2 & 3 & 4 \\
\hline Head & no response & raised & turned to one side & turned to both sides \\
Body & no response & sits up & moves or rears without changing location & walking \\
Arrest & no response & arrest & & \\
Groom & no response & groom & & \\
\hline
\end{tabular}

Note-Table is modified from Kфhler, 1976; a minimum score of 5 and maximum of 12 was the criteria for calling a response "orienting." 


\section{Behavioral OR}

The majority of orienting responses were search responses $(96 \%)$ consisting of either head or body movements, or a combination of these. Only three responses consisted of the arrest component alone, and two consisted of a combination of arrest and search components.

The behavioral responses were easy to observe. Responses to the initial stimuli were strong and distinct and repeated stimulus presentations led to gradual habituation (Figure 1). Fifty-five percent of the animals oriented during Trials $1-10$, whereas only $2 \%$ oriented during Trials 41-50.

The mean size of the OR during the first 25 trials was $6.10(\mathrm{SD}= \pm 1.16)$ and during the last 25 trials, $5.47(\mathrm{SD}= \pm .83)$, with a score of 5.00 being the minimum and 12.00 the maximum for calling a response "orienting." The difference between these is significant at the .025 level $[\mathrm{t}(115)=2.03]$.

\section{HR Responses}

Cardiac responses consisted of accelerations and decelerations. Only one animal reacted to one stimulus (Trial 1) with a HR decrease followed by an increase. Of all HR responses, $76 \%$ were accelerations and $23 \%$ were decelerations.

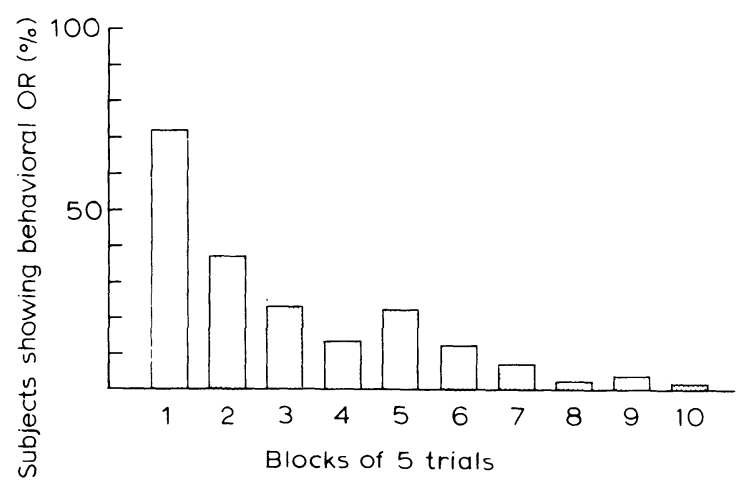

Figure 1. Percent of subjects showing behavioral orienting response (OR) across 50 trials.

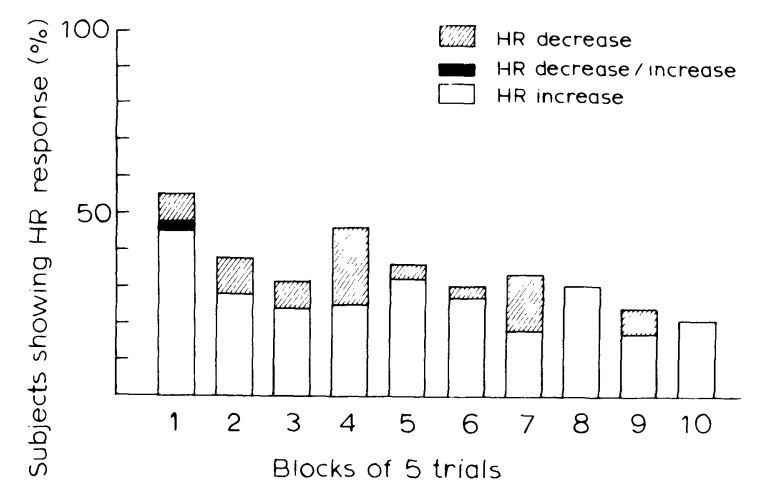

Figure 2. Percent of subjects showing heart rate (HR) increase, decrease, or decrease/increase responses across 50 trials.
Table 2

Mean Size of Cardiac Responses (in Beats per Minute) in Relation to Trials and Response Latency

\begin{tabular}{lcccrr}
\hline & \multicolumn{2}{c}{ HR Increase } & & \multicolumn{2}{c}{ HR Decrease } \\
\cline { 2 - 3 } & Mean & SD & & Mean & SD \\
\hline Trial 1-25 & 39.6 & 16.2 & & $39.2^{*}$ & 14.9 \\
Trial 26-50 & 37.3 & 14.1 & & $29.8^{*}$ & 7.3 \\
Short Latency & 37.1 & 15.1 & & $41.7^{* *}$ & 16.3 \\
Long Latency & 41.2 & 15.6 & & $33.1^{* *}$ & 11.1 \\
\hline
\end{tabular}

Note $-H R=$ heart rate; short latency $=1-50$ poststimulus beats; long latency $=51-100$ poststimulus beats; $S D=$ standard deviation. $\quad{ }^{*} p<.05 . \quad{ }^{* *} p<.025$.

The cardiac response showed a degree of habituation (Figure 2), although this was less pronounced than habituation of the behavioral OR. Forty-seven percent of the animals reacted with a cardiac response during Trials $1-10$ and $23 \%$ continued with the heart response during Trials 41-50. In addition, there was no relationship between the direction of HR change and the number of stimulus presentations. Seventyfour percent of the cardiac responses were accelerations during Trials $1-25$, and $80 \%$ were accelerations during Trials 26-50. Likewise, $26 \%$ of the responses were decelerations during Trials $1-25$, and $20 \%$ were decelerations during Trials 26-50.

Cardiac decelerations occurring during the first 25 trials were significantly larger than decelerations occuring during the last 25 trials $[\mathrm{U}(17,8)=39, \mathrm{p}=.05]$. Accelerations did not show such habituation (Table 2). Likewise, decelerations occurring within the first 50 poststimulus beats (short latency) were significantly larger than decelerations occurring during Poststimulus Beats 51-100 (long latency) $[\mathrm{U}(9,16)=$ $32.5, \mathrm{p}<.025$ ] (Table 2).

\section{HR and Behavioral OR}

No consistent relationship was found between the occurrence of the behavioral OR and the direction of HR change (Figure 3). However, some form of cardiac response was exhibited more often when an OR was present than when it was not $\left[\chi^{2}(1)=25.68\right.$, $p<.0005$ ]. The relative frequency of the various cardiac responses was about the same whether an OR occurred or not (Table 3). No differences were found between the number of cardiac responses occurring with or without an OR when latency of the cardiac response is considered (Table 3 ).

The mean size of ORs occurring with cardiac accelerations was $6.40(\mathrm{SD}= \pm 1.35)$, whereas the mean size of ORs occurring with decelerations was 5.78 $(\mathrm{SD}= \pm 1.30)$ and that without an accompanying $\mathrm{HR}$ response, $5.72(\mathrm{SD}= \pm .83)$. The difference between the size of ORs accompanied by cardiac acceleration and ORs occurring without a heart response, but not between ORs accompanied by accelerations and decelerations, was significant at the .05 level $[t(41)=$ 


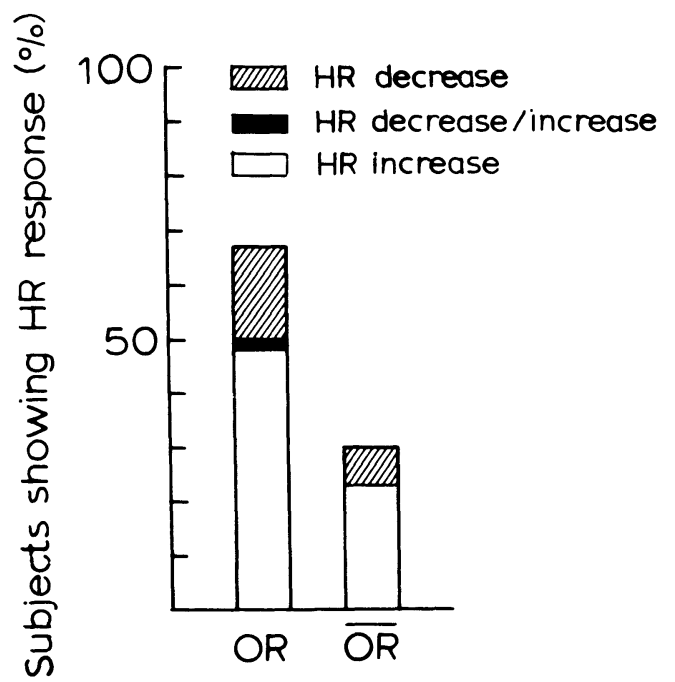

Figure 3. Percent of subjects showing heart rate (HR) increase, decrease, or decrease/increase responses when a behavioral orienting response is present (OR) or absent $(\overline{\mathrm{OR}})$.

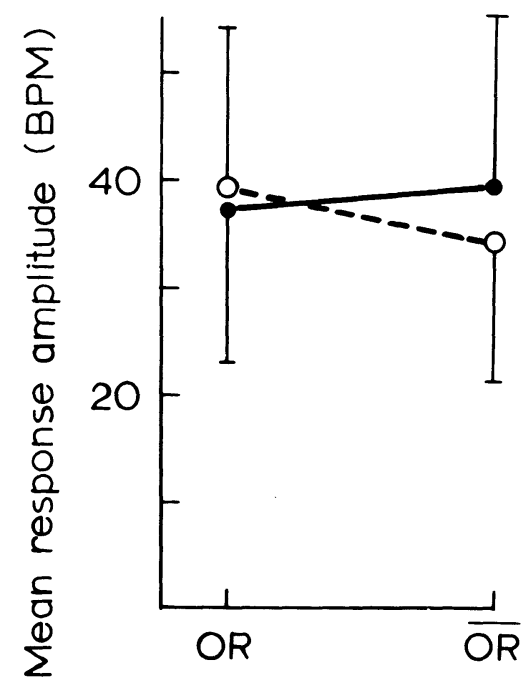

Figure 4. Mean amplitude (in beats per minute) of heart rate (HR) increase and decrease responses when a behavioral orient-

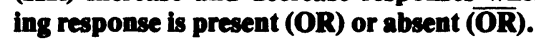

1.89]. Size of the OR did not correlate with the size of the cardiac response (rho $=.31$ and .10 for ORs occurring concomitantly with heart accelerations and decelerations, respectively). The only OR consisting of the arrest component alone and also accompanied by a cardiac response was followed by a 72-beat acceleration.

There were no differences in size of HR accelerations and decelerations when an OR occurred and when an OR did not occur (Figure 4). Nor were there any differences in the size of the HR response occurring without an OR during the first 25 trials and that occurring during the last 25 trials (Table 4). Because the OR habituated, during Trials 26-50 there were too few responses that were also accompanied by a cardiac response to allow for statistical analysis of habituation of HR responses occurring with ORs.

Of the short-latency (1-50 poststimulus beats) cardiac responses occurring with an $\mathrm{OR}$, decelerations were significantly larger than accelerations $[\mathrm{U}(16,4)$ $=12, p<.05]$ (Table 4). There were no further differences in size of the HR response with respect to orienting and response latency.

\section{DISCUSSION}

The behavioral criteria successfully delineated an orienting response that habituates both with respect to frequency and amplitude. The accompanying HR response does not appear to relate unequivocally to the behavioral OR. In fact, little evidence for the existence of a "cardiac OR" in the rat is found. In addition to the logical difficulties inherent in the concept of orienting with the heart, the prolonged occurrence of cardiac responses in spite of habituation of the behavioral OR is also seriously inadequate to support this concept.

It could be argued that the persistent cardiac responses represent a nonhabituating defense response

Table 3

Percent of Heart Responses in Relation to the Behavioral Orienting Response (OR) and Response Latency

\begin{tabular}{lcccccc}
\hline & \multicolumn{2}{c}{ HR Increase } & HR Decrease & \multicolumn{2}{c}{ Total } \\
\hline OR & 74 & & 26 & 100 & \\
Short Latency & & 46 & & 11 & & 57 \\
Long Latency & & 29 & & 14 & & 43 \\
OR & 78 & & 22 & & 100 & \\
Short Latency & & 49 & & 7 & & 56 \\
Long Latency & & 28 & & 16 & & 44 \\
\hline
\end{tabular}

Note $-H R=$ heart rate $; \overline{O R}=$ orienting response absent; short latency $=1.50$ poststimulus beats; long latency $=51.100$ post stimulus beats.

Table 4

Mean Size of Cardiac Responses (in Beats per Minute) in Relation to Behavioral Orienting Response (OR), Trials, and Response Latency

\begin{tabular}{|c|c|c|c|c|}
\hline & \multicolumn{2}{|c|}{ HR Increase } & \multicolumn{2}{|c|}{ HR Decrease } \\
\hline & Mean & SD & Mean & SD \\
\hline & \multicolumn{2}{|c|}{ OR } & \multicolumn{2}{|c|}{ OR } \\
\hline Trial $\quad 1-25$ & 38.2 & 14.3 & 41.3 & 14.2 \\
\hline Trial 26-50 & $26.1^{*}$ & 4.4 & $22.7 * *$ & \\
\hline Short Latency & $35.0 \dagger$ & 12.9 & $43.8 \dagger$ & 14.5 \\
\hline \multirow[t]{2}{*}{ Long Latency } & 40.8 & 16.1 & 35.6 & 15.4 \\
\hline & \multicolumn{2}{|c|}{$\overline{\mathrm{OR}}$} & \multicolumn{2}{|c|}{$\overline{\mathrm{OR}}$} \\
\hline Trial 1-25 & 41.0 & 18.0 & 37.4 & 16.1 \\
\hline Trial 26-50 & 38.0 & 14.2 & 30.8 & 7.3 \\
\hline Short Latency & 38.1 & 16.1 & 40.0 & 19.2 \\
\hline Long Latency & 41.5 & 15.8 & 32.0 & 9.3 \\
\hline
\end{tabular}

Note $-H R=$ heart rate $; \overline{O R}=$ orienting response absent $;$ short latency $=1.50$ poststimulus beats; long latency $=51.100$ post stimulus beats, $S D=$ standard deviation. $\quad{ }_{n}^{*}=2 . \quad *_{n}^{*}=1$. $t p<.05$. 
due to the general intensity (maximum $85 \mathrm{~dB}$ ) of the stimulus, or that a defense response has replaced orienting because of the aversiveness to which the animal is subjected by prolonged stimulation without the possibility of escape. Both arguments find little support. First, conclusive evidence as to what intensity is sufficient to produce defense cannot be found in the literature. The only condition that does find general support is that a fast rise time can produce startle if the minimum intensity reached is $90 \mathrm{~dB}$. Neither of these conditions were fulfilled in our experiment. Second, Sokolov's (1963) claim that prolonged and unavoidable stimulation can produce defense, which increases rather than decreases with repeated presentations (Thompson \& Spencer, 1966), is not supported in the present experiment, in which neither cardiac acceleration nor deceleration increased significantly in either size or frequency. Cardiac decelerations, especially responses with a short latency, habituated with respect to amplitude but not frequency and only partially fulfilled the prerequisite that an OR should habituate with repeated stimulation. When decelerations are considered in the context of the behavioral OR, too few responses prevent any conclusions from being drawn. The relative frequency of decelerations was unaffected by the number of stimulus presentations, either in relation to the OR or not. In addition, decelerations accounted for only $26 \%$ of observed unidirectional cardiac responses accompanying the behavioral response and can hardly be said to coincide with the OR enough to be called its correlate.

The predominance of the search component of the OR led us to expect that the accompanying heart responses would show more accelerative activity than the remaining heart responses simply because of the somatic requirements inherent in the behavioral response. In fact, nearly three-quarters of the heart responses accompanying the OR were accelerations. There was, however, a degree of decelerative activity, primarily with a short response latency, that "overrode" the somatic conditions expected to cause heart rate acceleration; in one case, the cessation of somatic activity resulted in a large cardiac acceleration. In addition, there was a certain amount of accelerative activity that was unrelated to the occurrence of the behavioral OR, suggesting that our criteria relied more on gross than on fine movements, or that unobservable muscle innervations, such as isometric contractions, had occurred. Responses such as fear or distress can affect the autonomic nervous system, causing withdrawal or vagal inhibition and sympathetic discharge and, thereby, heart acceleration beyond the somatic requirement in humans (Strømme, Wikeby, Blix, \& Ursin, 1978).
The heart response during orienting appears to be a concomitant response that is neither dependent on nor specific to the behavioral $O R$. We agree with Obrist et al. (1979) that at least in the rat the cardiovascular system is not a "crystal ball that provides insight into behavioral processes concerning orienting" (p. 220). How the organism reacts to a new stimulus in the environment can influence how the heart responds, but the consequences for the cardiovascular system are much more complex than have been assumed and do not follow simple notions of inhibition and facilitation.

\section{REFERENCES}

Germana, J., \& Klein, S. B. The cardiac component of the orienting response. Psychophysiology, 1968, 4, 324-328.

Graham, F. K., \& Clifton, R. K. Heart-rate change as a component of the orienting response. Psychological Bulletin, 1966, 65, 305-320.

Graham, F. K., \& Slaby, D. A. Differential heart rate changes to equally intense white noise and tone. Psychophysiology, $1973,10,347-362$.

Hoffman, H. S., \& Ison, J. R. Reflex modification in the domain of startle: I. Some empirical findings and their implications for how the nervous system processes sensory input. Psychological Review, 1980, 87, 175-189.

KøнLer, C. Habituation of the orienting response after medial and lateral septal lesions in the albino rat. Behavioral Biology, 1976, 16, 63-72.

LACEY, J. I., \& LACEY, B. C. The relationship of resting autonomic activity to motor impulsivity. In The brain and human behavior (Proceedings of the Association for Research in Nervous and Mental Disease). Baltimore: Williams \& Wilkins, 1958.

LyNN, R. Attention, arousal and the orientation reaction. Oxford: Pergamon Press, 1966.

Obrist, P. A., Langer, A. W., Light, K. C., Grignolo, A., \& McCubbin, J. A. Myocardial performance and stress: Implications for basic and clinical research. In H. D. Kimmel, E. H. van Olst, \& J. F. Orlebeke (Eds.), The orienting reflex in humans. Hillsdale, N.J: Erlbaum, 1979.

Pavlov, I. P. Conditioned reflexes. Oxford: Clarendon Press, 1927.

Roessler, R., Collins, F., \& Burch, N. R. Heart rate response to sound and light. Psychophysiology, 1969, 5, 359-369.

Soxolov, E. N. Perception and the conditioned reflex. Oxford: Pergamon Press, 1963.

Strømme, S. B., Wikeby, D. C., Blix, A. S., \& Ursin, H. Additional heart rate. In H. Ursin, E. Baade, \& S. Levine (Eds.), Psychobiology of stress: A study of coping men. New York: Academic Press, 1978.

Thompson, R. F., \& Spencer, W. A. Habituation: A model phenomenon for the study of neuronal substrates of behavior. Psychological Review, 1966, 73, 16-43.

Uno, T., \& Grings, W. W. Autonomic components of orienting behavior. Psychophysiology, 1965, 1, 311-321.

Ursin, H., Wester, K., \& Ursin, R. Habituation to electrical stimulation of the brain in unanesthetized cats. Electroencephalography and Clinical Neurophysiology, 1967, 23, 41-49.

(Manuscript received May 20, 1983; revision acçepted for publication October $3,1983$. 